\title{
Assessment of rapid ELISA test for detection of Epstein-Barr virus infection
}

\author{
B A Matheson, S M Chisholm, D O Ho-Yen
}

\begin{abstract}
$A$ rapid test for the detection of $\operatorname{IgM}$ and IgG Epstein-Barr nuclear antigen (EBNA-1) has been extensively marketed. If IgM to Epstein-Barr viral capsid antigen (EBV VCA) is taken as evidence of current EBV infection, one observer detected 17 of 38 such samples and the other 22 of 38 as acute. The positive predictive value of the test was $63 \%$, and the greatest difficulty was posed by the detection of IgM EBV VCA positive, heterophile antibody negative samples. Significant false positive results were obtained in sera with evidence of current Toxoplasma gondii, cytomegalovirus, and adenovirus infection. Rheumatoid factor was not a problem. Modification of the test protocol improved its performance: the positive predictive value rose to $87 \%$ and the negative predictive value to $81 \%$. Although our modifications did not increase the speed of the test, there was reliable information on the EBNA-1 state. The test is best used as an adjunct to other EBV serology, and laboratories should be aware of the limitations of the rapid test.
\end{abstract}

Epstein-Barr virus (EBV) is a prevalent, worldwide infection of which the most common clinical manifestation is infectious mononucleosis. An accurate diagnosis of infectious mononucleosis, which should be distinguished from "glandular fever", depends on finding appropriate, clinical, haematological and serological evidence of the disease. ${ }^{12}$ In Britain some clinicians have found it more convenient to obtain the haematological and serological evidence from haematology laboratories. Many haematology laboratories have therefore offered rapid tests for infectious mononucleosis, usually a modified PaulBunnell-Davidsohn (PBD) test, as part of their service. It has also been suggested that if there are sufficient Downey lymphocytes serological evidence of infectious mononucleosis is unnecessary. ${ }^{3}$

One drawback of the PBD test is that it measures heterophile antibody. At least $10 \%$ of patients with acute infectious mononucleosis do not form heterophile antibody and this figure is much higher in children. ${ }^{2}$ Tests such as specific IgM for EBV viral capsid antigen (VCA) have been the standard for virology laboratories. ${ }^{2}$ Recently, a rapid, synthetic peptide-based enzyme linked immunosorbent assay (ELISA) to detect antibodies to
Epstein-Barr nuclear antigen (EBNA) has been assessed ${ }^{4}$ and marketed (Ortho Diagnostics Systems, New Jersey, USA). We are aware that this test has been accepted by many haematology and other laboratories, but we feel that it should be independently assessed. In particular, it should not be compared with the PBD test, but with the IgM EBV VCA. This report documents our experience with it.

\section{Methods}

Each sample of serum sent to the Virology Laboratory, Raigmore Hospital, Inverness, was from an individual patient. Three groups with known IgM EBV VCA (Gull, Utah) and PBD results were chosen: 25 sera were IgM VCA positive, PBD positive; 13 sera were IgM VCA positive, PBD negative; and 34 sera were IgM VCA negative, PBD negative. Five control groups of sera were also tested: five sera with rubella IgM (Abbott, Wokingham, Berkshire), five sera with high titre adenovirus by the complement fixation test (PHLS Division of Microbiological Reagents, Colindale), nine sera with cytomegalovirus IgM (Gull, Utah), 10 sera with Toxoplasma gondii IgM (Organon, Netherlands) and 10 sera with high titre rheumatoid factor by nephelometry. Sera in these five control groups were $\operatorname{IgM}$ VCA negative, PBD negative.

RAPID ELISA

All the sera were tested in the rapid ELISA system for anti-EBNA (Monolert, Ortho Diagnostic Systems, New Jersey, USA) according to the manufacturer's instructions. Essentially, equal volumes of serum and washing buffer are added to both wells in plastic paddles coated with a synthetic peptide sequence of EpsteinBarr Nuclear Antigen (EBNA-1); paddles are incubated for two minutes at room temperature and then washed twice. Monoclonal antihuman IgG and IgM conjugated to horse radish peroxidase are added to appropriate wells, incubated, and washed as before. The substrate is added, incubated for two minutes at room temperature and stopped with $1 \%$ sodium dodecyl sulphate. Colour intensity in both wells (one IgG, one IgM) are read visually and compared: IgM > IgG (= acute infection); IgG > IgM (= past infection, immune); and no colour in both wells (= nonimmune). The results were read by two observers and compared.

\section{MODIFIED METHOD}

The rapid ELISA method was modified: equal 
Table 1 Performance of monolert rapid ELISA for anti-EBNA on test sera

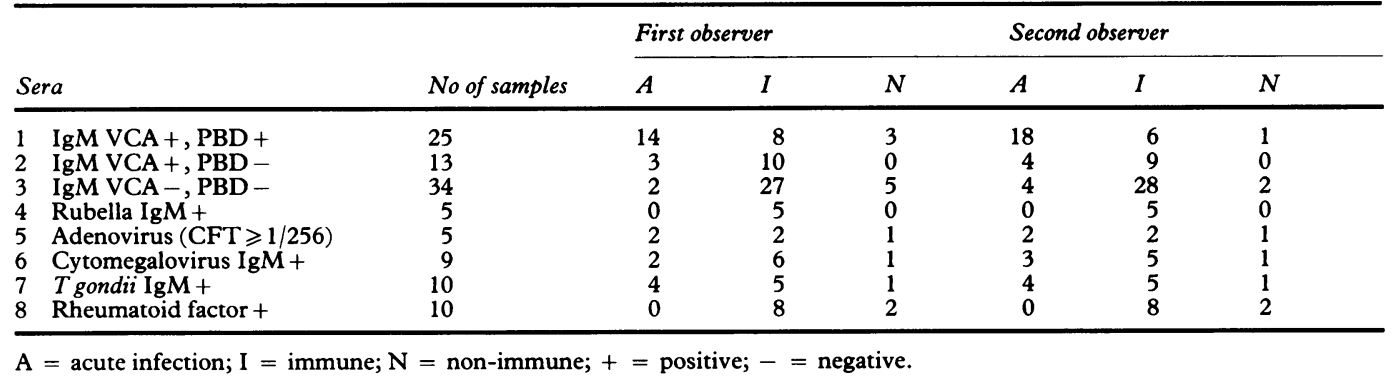

Table 2 Performance of modified method for anti-EBNA on test sera

\begin{tabular}{|c|c|c|c|c|c|c|c|c|}
\hline \multirow{2}{*}{\multicolumn{2}{|c|}{ Sera }} & \multirow[b]{2}{*}{ No of samples } & \multicolumn{3}{|c|}{ Visual } & \multicolumn{3}{|c|}{ Instrument } \\
\hline & & & $A$ & $I$ & $N$ & $A$ & $I$ & $N$ \\
\hline $\begin{array}{l}1 \\
2 \\
3 \\
4 \\
5 \\
6 \\
7\end{array}$ & $\begin{array}{l}\text { IgM VCA }+, \text { PBD + } \\
\text { IgM VCA +, PBD - } \\
\text { IgM VCA -, PBD - } \\
\text { Rubella IgM+ } \\
\text { Adenovirus }(\text { CFT } \geqslant 1 / 256) \\
\text { Cytomegalovirus IgM }+ \\
\text { Tgondii IgM + }\end{array}$ & $\begin{array}{r}23 \\
13 \\
22 \\
5 \\
2 \\
8 \\
8\end{array}$ & $\begin{array}{r}22 \\
5 \\
4 \\
0 \\
2 \\
4 \\
4\end{array}$ & $\begin{array}{r}1 \\
8 \\
18 \\
5 \\
2 \\
4 \\
4\end{array}$ & $\begin{array}{l}0 \\
0 \\
0 \\
0 \\
0 \\
0 \\
0\end{array}$ & $\begin{array}{r}21 \\
5 \\
3 \\
0 \\
0 \\
1 \\
0\end{array}$ & $\begin{array}{r}2 \\
8 \\
18 \\
5 \\
4 \\
6 \\
8\end{array}$ & $\begin{array}{l}0 \\
0 \\
1 \star \\
0 \\
0 \\
1 \\
0\end{array}$ \\
\hline
\end{tabular}

$\mathrm{A}=$ acute infection; $\mathrm{I}=$ immune; $\mathrm{N}=$ non-immune; ${ }^{\text {borderline }} \mathrm{I} / \mathrm{N} ;+$ = positive; - = negative.

volumes of the patient's serum and washing buffer were added to tubes and vortexed before being added to the wells of the paddles; the incubation times of the first two stages were extended to 60 minutes; the colour intensity was read visually two minutes after the substrate was added; the reaction was stopped after 15 minutes; and the contents of each well were transferred to a microtitre plate and the absorbances determined by spectrophotometry (Dynatech MR600).

\section{Results}

The use of wells in plastic paddles was novel and made the washing procedure easy. The manufacturer's instructions were clear and easy to follow. The performance of the Monolert, according to the manufacturer's instructions, is documented in table 1. There were great interobserver differences: of the $\mathbf{7 2}$ EBV positive and negative sera, there were disagreements in 16 results, but of the 39 control sera, there were disagreements in only two. More importantly, of 38 IgM VCA positive sera, the first observer detected 17 and the other 22, and of the 73 IgM VCA negative sera, the first observer recorded 10 and the second 13 as acute infection. Although the test was simple to perform, these results would not allow us to adopt the test routinely.

When modified with one hour incubation periods, there was an improvement in the visual results of the IgM VCA positive sera (table 2), but still 14 of 47 negative samples were reported as acute infection. Assessment of the optical density results allowed the following criteria to be established: a standard G:M ratio was obtained by averaging the three highest G:M ratios of the VCA positive, PBD positive group. A standard $M$ value was calculated by averaging the $M$ absorbance values in the VCA negative, PBD negative group, and a standard $G$ value was obtained by averaging the $G$ absorbance values in the VCA positive, PBD positive group. The standards were then used as follows: ( < standard G:M ratio, > standard $M$ absorbance) $=$ acute infection; ( $>$ standard $\mathrm{G}: M$ ratio, $>$ standard $\mathrm{G}$ absorbance) $=$ immune; and (< standard $M$ absorbance, < standard $\mathrm{G}$ absorbance) = non-immune.

With these criteria (table 2), all but two of the IgM VCA positive and PBD positive samples were detected, and all but three of the IgM VCA negative, PBD negative samples were assessed as not acute infection. The difficulty was that only five out of 13 of IgM VCA positive, PBD negative samples were detected as acute infection. The criteria dramatically reduced the false positive results among the samples which were positive for other infections: only one of the 25 samples CMV IgM was positive. The sensitivity, specificity, positive and negative predictive values of the methods using different criteria of assessment are shown in table 3.

Table 3 Percentage, specificity, positive and negative predictive values of test for anti-EBNA compared with that for IgM anti-EBV VCA

\begin{tabular}{|c|c|c|c|c|c|}
\hline & \multicolumn{3}{|c|}{ Original visual method } & \multicolumn{2}{|c|}{ Modified method } \\
\hline & Observer (1) & Observer (2) & Average & Visual & Instrument \\
\hline $\begin{array}{l}\text { Sensitivity } \\
\text { Specificity } \\
\text { Positive predictive value } \\
\text { Negative predictive value }\end{array}$ & $\begin{array}{l}44 \\
86 \\
63 \\
75\end{array}$ & $\begin{array}{l}58 \\
82 \\
63 \\
79\end{array}$ & $\begin{array}{l}51 \\
84 \\
63 \\
77\end{array}$ & $\begin{array}{l}75 \\
70 \\
66 \\
78\end{array}$ & $\begin{array}{l}72 \\
91 \\
87 \\
81\end{array}$ \\
\hline
\end{tabular}




\section{Discussion}

The rapid ELISA test described is marketed as a test for infectious mononucleosis, but it is more correctly a test for Epstein-Barr virus (EBV) infection. The test is based on peptide 62, an Epstein-Barr nuclear antigen (EBNA1), coated on to the solid phase. The initial good performance of the test ${ }^{4}$ and the subsequent adaptation to a rapid test ${ }^{5}$ (six minutes) was impressive, and it is understandable that the test has been adopted by many laboratories, but our results show that this test, in its present form, has serious limitations.

The visual assessment of the colour change contributes to the speed of the test. Unfortunately, there are great observer differences in determining the intensity of colour. The decision may be further complicated when many samples are being tested as each paddle has to be assessed separately, and the manufacturers state that comparisons between paddles should not be made. Although the paddle system is clever and useful, if a few samples are being tested the system becomes cumbersome when there are many samples.

It has been suggested that the rapid ELISA is more sensitive than the PBD as it can detect EBV infection which is heterophile antibody negative. ${ }^{5}$ We found that the rapid ELISA detected less than half of such samples, and with the PBD positive samples there was a failure to detect $44 \%$ by one observer and $28 \%$ by another. Thus in our hands the positive predictive value of the test when compared with IgM EBV VCA was $63 \%$. Ironically, the very samples for which the manufacturers claim an advantage for the rapid ELISA, IgM VCA positive, PBD negative, are the ones which the rapid ELISA has most difficulty identifying. When compared with the IgM EBV VCA, which is generally accepted to be the best test for diagnosing EBV infection, ${ }^{2}$ the rapid ELISA did not do well.

The rapid ELISA also lacked specificity. Previous assessments ${ }^{45}$ had not tested many serum samples that were positive for other infections. The rapid ELISA produced many false positive results (table 1 ) in infections which may be readily confused with infectious mononucleosis clinically. The manufacturer's instructions only mention cross reactivity with cytomegalovirus infection. We have confirmed this cross reactivity, but we have also shown others; and the negative predictive value of the test was $77 \%$. Although we agree that rheumatoid factor does not interfere with the rapid ELISA test, this is only a small advantage.

When we increased the incubation times of the test and read the results objectively, criteria could be established that improved the performance of the test. We subsequently realised that the original assessment of the technique ${ }^{4}$ was similar to ours and the results comparable. With our modification, the positive predictive value was $87 \%$ and the negative predictive value $81 \%$. While our modification does not have the advantage of a rapid test, it does provide information on EBNA-1 specific antibody. We believe that especially where other virus serology is not done, laboratories must be aware of the severe limitations of the rapid ELISA.

We are grateful to Miss V MacFarquhar for secretarial assistance and to the Tayside Post Viral Fatigue Syndrome Support Group for a grant.

1 Ho-Yen DO, Martin KW. The relationship between atypical lymphocytosis and serological tests in infectious mononucleosis. J Infect 1981;3:324-31.

2 Ho-Yen DO. Infectious mononucleosis. Update 1984;28:184-92.

3 Ho-Yen DO. Is the serological diagnosis of infectious mononucleosis always necessary? Br Med J 1983;287:1187-9.

4 Geltosky JE, Smith RS, Whalley A, Rhodes G. Use of a synthetic peptide-based ELISA for the diagnosis of infectious mononucleosis and other diseases. J Clin Lab Anal 1987;1:153-62.

5 Levin M, Rhodes G, Sumaya C, et al. Diagnosis of infectious mononucleosis using a rapid ELISA which detects IgM antibody to an Epstein-Barr virus nuclear antigen (EBNA) peptide. Abstracts. Interscience Conference on Antimicrobiol Agents and Chemotherapy. Washington, DC: American Society for Microbiology, 1988. 